\title{
Optical Absorption Coefficient on-center donor impurity in a spherical core/shell quantum dots
}

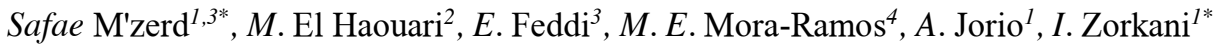 \\ ${ }^{1}$ Group: "Nanomaterials and Renewable Energies"LPS, Faculté des sciences Dhar Mehraz, Fes - Morocco \\ ${ }^{2}$ Centre Régional des Matières de l'Éducation et de Formation (CRMEF), Tanger, Morocco.Group of Optoelectronic of Semiconductors \\ and Nanomaterials, ENSET, Mohammed V University in Rabat, Morocco. \\ ${ }^{3}$ Group of Optoelectronic of Semiconductors and Nanomaterials, ENSET, Mohammed V University in Rabat, Morocco. \\ ${ }^{4}$ Centro de Investigacion en Ciencias-IICBA, Universidad Autonoma del Estado de Morelos, Av. Universidad 1001, CP 62209, \\ Cuernavaca, Morelos, Mexico
}

\begin{abstract}
The polaronic effect on the linear, third-order nonlinear and total optical absorption coefficients have been calculated in the case of GaAs/AlAs core/shell quantum dot, with the impurity is positioned at the central radial position of the GaAs shell. The calculations are realized in the framework of the effective mass approximation and the numerical results are obtained by using a variational method and an infinite confining potential. The results show that the polaronic effect has a great influence on optical properties of core/ shell quantum dot, he causes a red-shift of the nonlinear optical coefficients associated to light absorption. Also, the polaronic effect is enhanced with the decreasing of quantum dot radius.
\end{abstract}

\section{Introduction}

The electronic and the optical properties in a semiconductor quantum dot structure are influenced by the presence of impurities, the physical properties related with impurity centers have been systematically investigated with the use different methods such as the variational approach[1-8], the tight-binding selfconsistent linear screening scheme[9], the strong confinement approximation[10-11], and the perturbation theory[12]. Using the potential morphing technique, the binding energies of hydrogenic impurity states confined in a quantum well with an external field are investigated by Baskoutas et al [13-14]. Furthermore, Zhu et al [15] investigated the binding energy of hydrogenic donor impurity in a spherical quantum dot using the linear variational method. Li et al [16] calculated the electronic states of a hydrogenic donor impurity in lowdimensional semiconductor nanostructures in the framework of effective-mass envelope-function theory by using the plane wave method. Recently, impurity states in core/shell with different potential have been studied as well [17-20].

The linear and nonlinear optical properties of semiconductor quantum wells and QDs have attracted much attention in recent either theoretically of with a practical view. The features of the optical absorption (OA) [9, 21-24] and refractive index change (RIC) [25] in these systems have the potential for device applications in far-infrared laser amplifiers[26], photodetectors [27], and high speed electro-optical modulators [1]. The linear and nonlinear optical absorptions in semiconductor superlattice systems were afterwards investigated by Shi et al.[28]. More recently, Unlu et al. [29], have analyzed the linear and nonlinear intersubband optical absorption coefficients and refractive index changes in a quantum box with finite confining potential, whilst the optical absorption and refractive index changes in a cylindrical QD were examinated by Liu et al [30].

As QDs are usually made of polar materials, the electron-LO phonon interaction must be taken into consideration for the appropriate description of electronic properties. Recently, a considerable number of theoretical studies on the electron-phonon effect on impurity energies in QDs in the presence of external fields have been performed [31-38]. M'zerd et al. have investigated the influence of the electron-LO-phonon interaction and of the position of a donor impurity atom on the linear and third-order nonlinear optical absorption and relative refractive index change coefficients in core/shell quantum dot [39].

In the present work, we are aimed at studying the Optical Absorption Coefficient associated to a hydrogenic donor states in GaAs-based inhomogeneous QD, so-called core/shell structure, taking into account the effect of the interaction with polar optical phonons. The article is organized in the following way: The model considered, the method used and the relations for OAC are presented in Section 2. Numerical results and related discussions

\footnotetext{
Corresponding author: safae.mzerd@gmail.com
} 
will be given in section 3 while the corresponding conclusions are presented in section 4 .

\section{Theoretical Framework}

\subsection{The electron-impurity plus LO-phonon Hamiltonian}

Let us consider a hydrogenic impurity located anywhere in the shell region of a core/shell quantum dot of the AlAs(core)/GaAs(shell)/AlAs (outer matrix) design. The confining potential is assumed to be an infinitely deep well -as depicted in Fig. 1- and the charge carrier is interacting with LO- phonons.

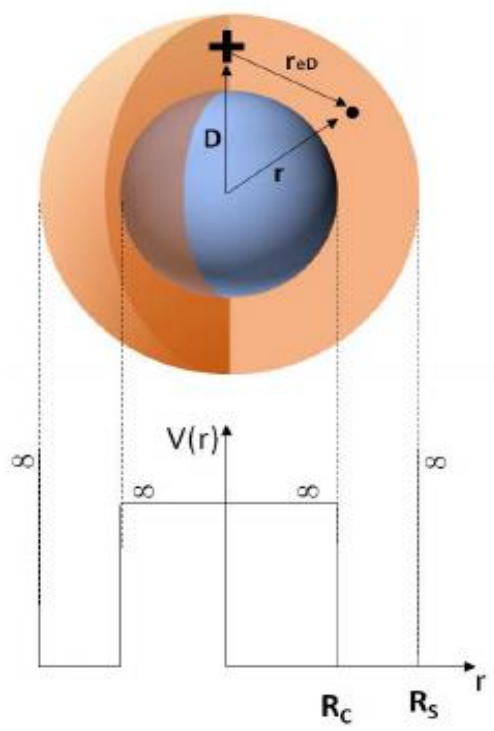

Fig. 1. Schematic diagram of core/shell Quantum Dot structure. $R_{C}$ and $R_{S}$ the core and the shell radius respectively

In the framework of the effective-mass approximation and using the Frohlich model for describing the electronLO phonon coupling, the Hamiltonian of the system is given by:

$$
H=H_{0}+H_{p h}+H_{e-p h}
$$

Here the electronic Hamiltonian is given by:

$$
H_{0}=-\frac{\hbar}{2 m_{\mathrm{ep}}^{8}} \nabla^{2}+V_{w}(r)+V(r)
$$

Where $\mathrm{V}_{\mathrm{w}}(\mathrm{r})$ is the confining potential :

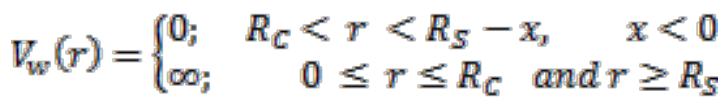

$\mathrm{V}(\mathrm{r})$ is the impurity-related Coulombic potential energy:
$V(r)=-\frac{e^{2}}{\varepsilon_{\infty} r_{\theta D}}$

Where $r_{e D}=|\vec{r}-\vec{D}|=\sqrt{r^{2}+D^{2}-2 r D \cos (\theta)}$ is the electron impurity distance, and $\theta$ is the angle between the electron $\vec{r}$, and the impurity vector position, $\vec{D}$.

The LO phonon Hamiltonian $\mathrm{H}_{\mathrm{ph}}$ is written as [40]:

$H_{p h}=\sum_{l m q} \hbar w_{L O}\left(a_{\operatorname{lm}}^{+}(q) a_{\operatorname{lm}}(q)+\frac{1}{2}\right)$

where $a_{l m}^{+}(q)$ and $a_{l m}(q)$ are, respectively, the creation and annihilation operators of a LO-phonon with quantum numbers $1, \mathrm{~m}$ and frequency $h w_{L O}$.

The electron-phonon interaction operator $H_{e-p h}$ is given within the well-known Frohlich continuum model adapted for this particular physical situation of an electron in a spherical environment. $H_{e-p h}$ depends on the coordinates of both impurity and electron, reflecting the fact that both of them, being charged, interact with the polar optical phonon polarization field. Using the LeeLow-Pines canonical transformation [41], Aldrich and Bajaj [42] have deduced an effective Hamiltonian $\mathrm{H}_{\text {eff }}$ for a Coulombic-coupled system, taking into account the interaction with LO-phonons:

$H_{e f f}=-\frac{\hbar}{2 m_{e p}^{*}} \nabla^{2}+V_{w}(r)+V(r)+H_{p h}^{t}$

Here $\mathrm{m}_{\mathrm{ep}}{ }^{*}$ denotes the so-called polaron (electronphonon-renormalized) effective mass, given by:

$m_{e p}^{*}=m_{e}^{*} \frac{(1+\alpha / 12)}{(1-\alpha / 12)}$

While the term $\mathrm{H}_{\mathrm{ph}}^{\mathrm{t}}$ is the renormalized phonon energy due to the interaction with the electron. Its expression for an off-center impurity in a quantum dot is given by:

$H_{p h}^{t}=-\frac{e^{2}}{2 \varepsilon^{\prime} r_{E D}} \exp \left(-\eta r_{E D}\right)+\frac{e \eta}{2 \varepsilon^{\prime}} \frac{\exp \left(-\eta r_{E D}\right)}{1+\alpha / 12+\alpha /(4+\alpha / 3)}-\alpha \hbar w_{L O}$

In these expressions, the appearing parameters are:

$$
\begin{aligned}
& \mathrm{\eta}=\left(\frac{2 m_{e}^{*} w_{L O}}{\hbar}\right)^{1 / 2} \\
& \alpha=\frac{e^{2} \eta}{2 \varepsilon^{\prime} \hbar w_{L O}}, \quad \frac{1}{\varepsilon^{\prime}}=\frac{1}{\varepsilon_{\infty}}-\frac{1}{\varepsilon_{0}}
\end{aligned}
$$

In this case, $\alpha$ is the electron-LO-phonon coupling coefficient and $\mathrm{m}_{\mathrm{e}}^{*}$ is the electron conduction band effective mass in the GaAs-shell region. The donor impurity ground state wave function is a solution of the Schrodinger equation: 


$$
H_{\text {eff }} \psi=E \psi
$$

In order to determine it we shall use the variational method to solve the Schrodinger-like effective mass equation. In accordance, the trial wave function of the $1 \mathrm{~s}$ state is chosen as [39]:

$$
\psi_{1 s}(r)=N_{1} \frac{\sin \left(\pi \frac{r-R_{C}}{R_{S}-R_{C}}\right)}{r} \exp \left(-\alpha r_{e D}\right)
$$

while for the $1 \mathrm{p}$-like excited state the trial wave function is taken as[39]:

$$
\psi_{1 p}(r)=N_{2}\left[\frac{\sin \left(\gamma \frac{r-R_{C}}{R_{S}-R_{C}}\right)}{(\gamma r)^{2}}-\frac{\cos \left(\gamma \frac{r-R_{C}}{R_{S}-R_{C}}\right)}{\gamma r}\right] \cos (\theta) r_{\mathrm{ED}} \exp \left(-b r_{\mathrm{ED}}\right)
$$

with $\mathrm{N}_{1}$ and $\mathrm{N}_{2}$ being the normalization constants, and $\gamma$ represents the second zero of the spherical Bessel function [39].

The first and second state energies are obtained by minimizing the following means values respectively with variational parameters $a$ and $b$ :

$$
E_{i}=\min \frac{\left\langle\psi_{i}\left|H_{e f f}\right| \psi_{i}\right\rangle}{\left\langle\psi_{i} \mid \psi_{i}\right\rangle}, i=(1 s, 1 p)
$$

\subsection{Linear, nonlinear and total Optical Absorption coefficients}

In this work, we limit ourselves to consider the optical transition between the ground (1s) and first excited (1p) states. The expressions of the linear and third-order nonlinear Optical Absorption coefficients can be obtained by a density matrix approach and a perturbation expansion method [22]. The first and third components of Optical Absorption coefficients are [43]:

$$
\beta(\omega, I)=\beta^{(1)}(\omega)+\beta^{(3)}(\omega, I)
$$

and the linear and third-order nonlinear parts of the OAC are, respectively:

$$
\beta^{(1)}(\omega)=\omega \sqrt{\frac{\mu}{\varepsilon}} \frac{h \Gamma_{f i}\left|M_{f i}\right|^{2} \sigma}{\left(E_{f i}-\hbar \omega\right)^{2}+\left(\hbar \Gamma_{f i}\right)^{2}}
$$

$$
\begin{aligned}
& \text { And } \\
& \begin{aligned}
\beta^{(3)}(\omega, I)= & -\omega \sqrt{\frac{\mu}{\varepsilon}}\left(\frac{I}{2 \varepsilon_{0} n_{r} c}\right) \frac{4 h \Gamma_{f i}\left|M_{f i}\right|^{4} \sigma}{\left[\left(E_{f i}-\hbar \omega\right)^{2}+\left(h \Gamma_{f i}\right)^{2}\right]^{2}} \times \\
& {\left[1-\frac{\left|M_{f f}-M_{i i}\right|^{2}}{4\left|M_{f i}\right|^{2}} \times \frac{3 E_{f i}^{2}-4 h \omega E_{f i}+h^{2}\left(\omega^{2}-\Gamma_{f i}^{2}\right)}{E_{f i}^{2}+\left(h \Gamma_{f i}\right)^{2}}\right] }
\end{aligned}
\end{aligned}
$$

In the former equations $E_{f i}=E_{f} E_{i}$ denotes the difference between the final and initial level energies, whilst $\mathrm{M}_{\mathrm{fi}}=$ $\left.\mathrm{e}<\psi_{\mathrm{i}}|\mathrm{r} \cos (\theta)| \psi_{\mathrm{f}}\right\rangle$ is the electric dipole moment of the transition between the states $i$ and $f$ with selection rule on the quantum number 1 given by $\Delta l= \pm 1$. In addition, we use the following notations for the remaining parameters: $c$ is the speed of light in vacuum, $\sigma$ is the electron density related to the occupied volume by the relation:

$$
\sigma=\frac{n}{V}=\frac{3 n}{4 \pi\left(R_{S}^{3}-R_{C}^{3}\right)}
$$

$\mathrm{I}$ is the intensity of the -linearly polarized- incident electromagnetic field, $\mu$ is the permeability of the system, $\mathrm{n}_{\mathrm{r}}$ is the semiconductor relative refractive index, $\varepsilon_{0}$ is the dielectric constant of the vacuum, $\hbar \omega$ is the incident photon energy; and $\hbar \Gamma_{\mathrm{fi}}=1 / \tau_{\mathrm{fi}}$ is the non-diagonal damping term associated with the relaxation rate between the final and initial states. $\hbar_{\mathrm{fi}}$ is defined as the inverse of the relaxation time $\tau_{\mathrm{fi}}$.

\section{Results and Discussion}

In this section, we will discuss the influence of polaronic corrections on the linear, third -order nonlinear, and total contributions to the optical absorption coefficient (OAC) in a spherical AlAs/GaAs core/shell according with the model outlined above. In Table 1 we present the values of the basic material input parameters considered in the calculation.

Table1: Physical parameters, of GaAs and AlAs. $\mathrm{R}_{0}$ and $\mathrm{a}_{0}$ are the donor energy and Bohr radius respectively.

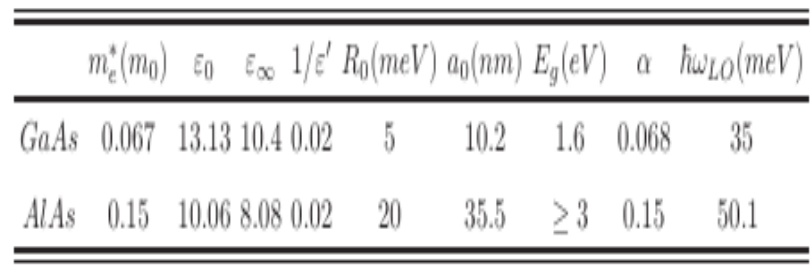

The Fig. 2 shows the variation of the transition energy $E_{f i}$ as a function of the width of the shell layer $R_{S}-R_{C}$ in a structure in which $\mathrm{R}_{\mathrm{C}}$ is fixed at $0.5 \mathrm{a}_{0}$. For the sake of illustration, we choose to discuss in detail the case in which the impurity is positioned at the central radial position of the GaAs shell. The transition energy goes from larger to smaller values as long as the thickness of the shell augments mainly due to the reduction in the degree of electron wave function localization inside the quantum well region. To justify this assertion just focus our attention on the curve that corresponds to the absence of phonon effects. Although the presence of the impurity center includes an attractive interaction that tends to lower the electron energy, even for small shell thickness, where the proximity of the electron and the impurity would lead to a stronger electrostatic coupling, the increase in the transition energy indicates that when $\left(R_{S}-R_{C}\right) \rightarrow 0$ there is a further separation between the state energies. The experience shows that the greater 
displacement -upwards- occurs for the $1 \mathrm{p}$ state whereas the ground state suffers a smaller shift. Then, for increasing values of the shell width, the reduction in the transition energy has, again, more to do with the fall in the energies of the two involved states, with a stronger reduction in the $1 \mathrm{p}$-like state level. This time, the electrostatic attraction plays an even lesser role provided the increment in the expected electron-impurity distance.

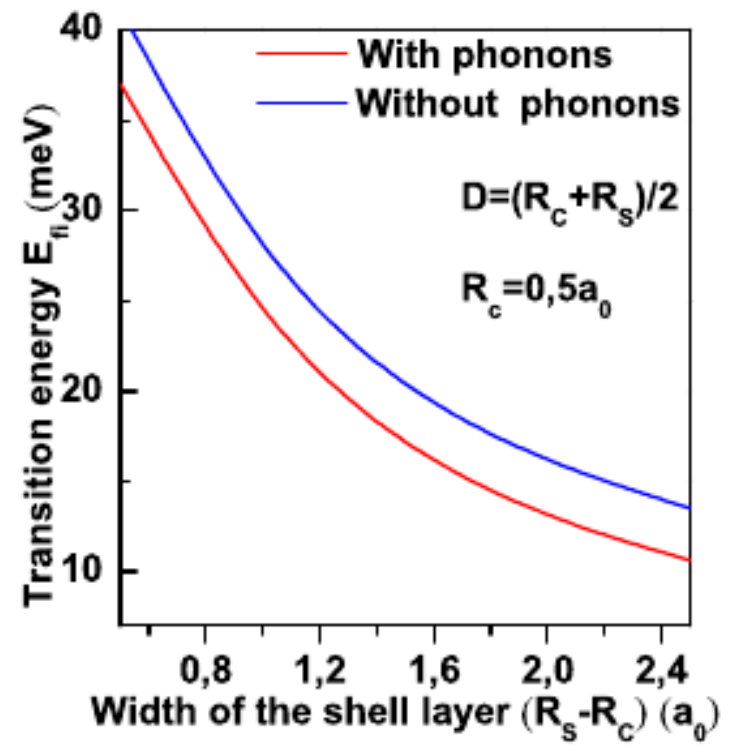

Fig. 2. Transition energy $E_{f i}=E_{T 1 p}-E_{T 1 S}$ as a function of the shell layer width $\left(\mathrm{R}_{\mathrm{S}}-\mathrm{R}_{\mathrm{C}}\right)$, with and without electron-phonon interaction contribution, with $\mathrm{D}=\left(\mathrm{R}_{\mathrm{S}}+\mathrm{R}_{\mathrm{C}}\right) / 2$.

On the other hand, when the influence of the electronphonon interaction is taken into account, the transition energies keep the decreasing behavior with $\left(\mathrm{R}_{\mathrm{S}}-\mathrm{R}_{\mathrm{C}}\right)$ but their values are all the way lower than those obtained without including the electron-phonon coupling. The reduction is less notorious in the interval of small values of the shell thickness and this effect has two main explanatory reasons. First, as one may see from Eq.(6), that the renormalized carrier mass is larger than the conduction band effective one. This implies a reduction in the transition energy. But, at the same time, the decrease in the shell size leads to the growth of this energy difference. This means that there is a competition between the two influences and the resulting lower values indicate that the mass-increasing effect seems to predominate. Moreover, when the difference $\left(R_{S}-R_{C}\right)$ augments, the further reduction of the transition energy difference has a clear dominance from the increment in the effective mass due to the polaronic effect. Indeed, one must not forget the contribution coming from the renormalized phonon energy of Eq. (7) which causes a shift downwards of the whole calculated spectrum.

For small enough values of $r_{e D}$, the negative term could prevail over the remaining two positive ones, thus adding an additional reduction of the electron energies. However, this part does not influence the transition energy difference because it is the same correction to both involved levels.
The variation of the off-diagonal component of the electric dipole moment associated with the transition between the $1 \mathrm{~s}$ - and $1 \mathrm{p}$-like states is shown as a function of the shell layer width is shown in Fig. 3. Considering that the squared absolute value of this quantity is the factor having an impact on the optical coefficients it is possible to conclude that $\left|\mathrm{M}_{\mathrm{fi}}\right|^{2}$ has, in this case, a mixed behavior that shows an initial increase and, for larger values of $\left(\mathrm{R}_{\mathrm{S}}-\mathrm{R}_{\mathrm{C}}\right)$, a reduction, which is slighter in the case where the electron-phonon interaction is included. In this latter case, the magnitude of the non-permanent polarization is significantly larger, which indicates a stronger overlapping of the involved wave functions owing to the higher carrier localization associated with the electron-phonon interaction.

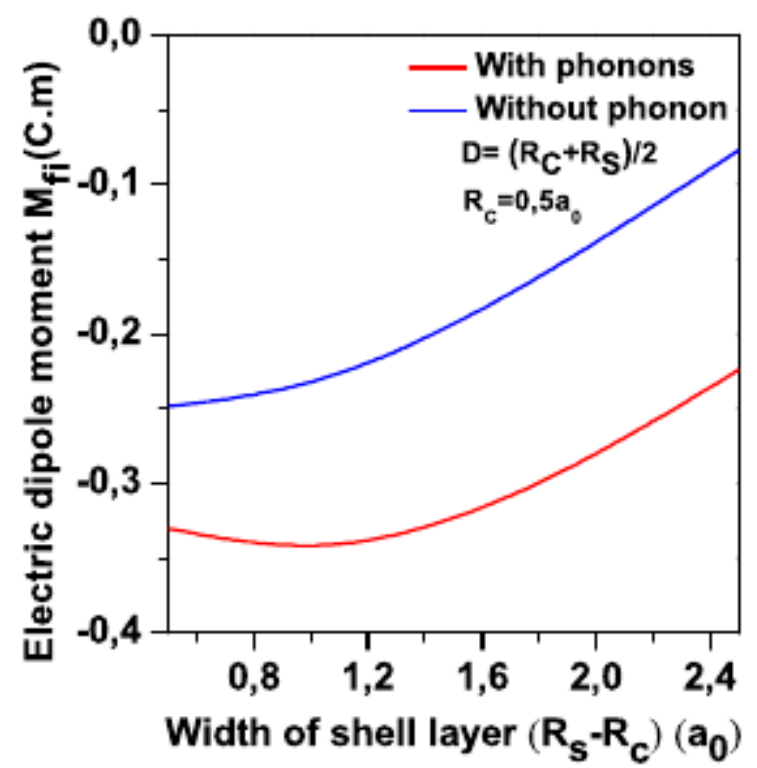

Fig. 3. Electric dipole Moment Mfi with and without phonon as a function of the shell layer width $\left(R_{S}-R_{C}\right)$, with $D=\left(R_{S}+R_{C}\right) / 2$.

The behavior of the OAC can be explained in straightforward manner by taking into account the features of both the transition energy difference and the electric dipole moment squared modulus matrix element. In what follows, we proceed to discuss these optical responses when the donor is localized on center the GaAs shell.

The Fig. 4 illustrates the linear, third-order nonlinear and total OACs, as functions of the incident photon energy for different $\mathrm{QD}$ radius, $\mathrm{R}_{\mathrm{S}}=1 \mathrm{a}_{0}$ and $\mathrm{R}_{\mathrm{S}}=2 \mathrm{a}_{0}$, with and without the inclusion of polaronic corrections. The single dopant is supposed to be placed at the center of the shell $\mathrm{D}=\left(\mathrm{R}_{\mathrm{S}}+\mathrm{R}_{\mathrm{C}}\right) / 2$. In addition, the nonlinear contributions are calculated assuming a value of $\mathrm{I}=200 \mathrm{MWm}^{-2}$ for the incident light intensity. 

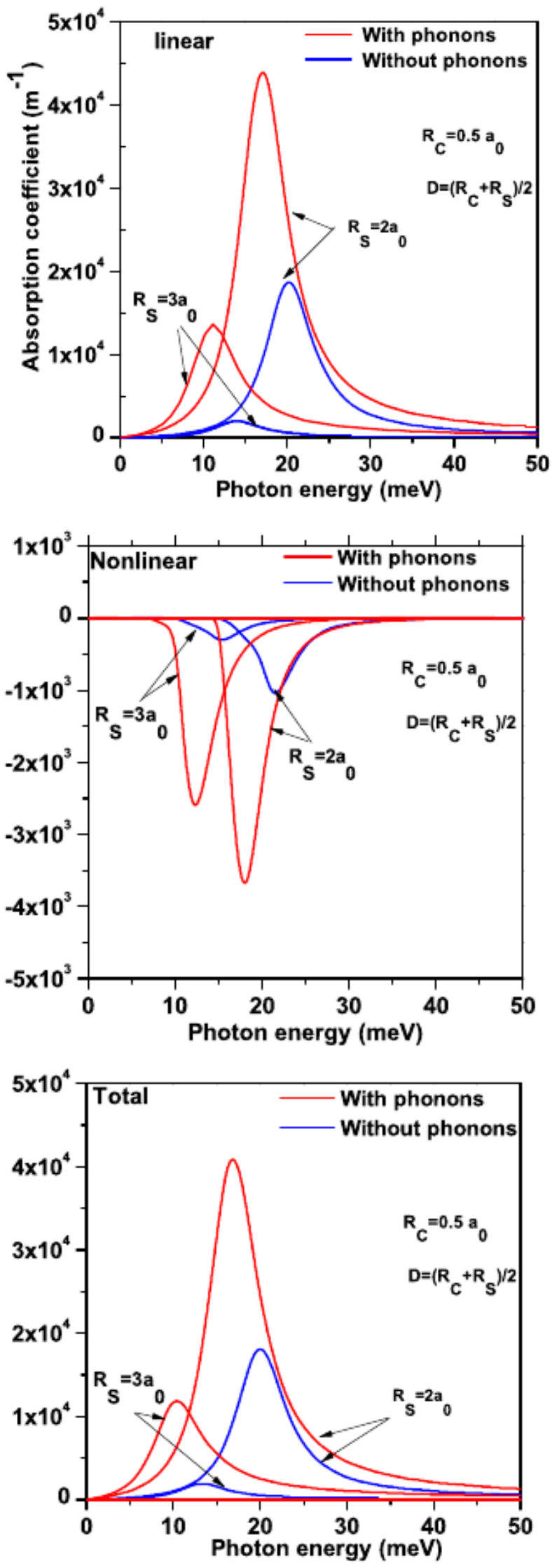

Fig. 4. The linear,third-order nonlinear and total Optical Absorption coefficients with phonon as a function of photon energy $\hbar \omega$ for different IQD radii $\mathrm{R}_{\mathrm{S}}=1 \mathrm{a}_{0}$ and $\mathrm{R}_{\mathrm{S}}=2 \mathrm{a} 0$, with $\mathrm{D}=$ $\left(\mathrm{Rs}_{\mathrm{S}}+\mathrm{R}_{\mathrm{C}}\right) / 2$.
The features of the OAC, in regard to the resonant peak or maxima and minima positions as well as with their amplitudes can be understood following the discussion made above for the transition energy difference and the $\left|\mathrm{M}_{\mathrm{fi}}\right|^{2}$ factor. As long as the value of $\mathrm{R}_{\mathrm{S}}$ diminishes (with Rc fixed), the signals become blue-shifted.

This also implies a growth of the OAC resonant peak amplitudes due to the combination of the increase in $\omega_{\mathrm{fi}}$ and the increment in the squared modulus of the electric dipole moment off-diagonal matrix element.

It can be readily noticed that he influence of the electronphonon interaction causes the overall red-shift of the optical responses, which is in accordance to the associated variation of $\mathrm{E}_{\mathrm{fi}}$ shown in Fig. 2, On the other hand, the polaronic effect reflects in a significant increment of the coefficient amplitudes, even in the OAC case where a diminishing value of $\omega_{\mathrm{fi}}$ is involved. Again, the explanation follows our comments on Fig. 3 above.

\section{Conclusions}

We have investigated the influence of the electron-LOphonon interaction when the donor is localized on center the GaAs shell on the linear and third-order nonlinear optical absorption coefficients in core/shell quantum dot of the $\mathrm{AlAs}(\mathrm{Core}) / \mathrm{GaAs}$ (well)/AlAs(outer matrix) type, assuming an infinite confining potential model. Our results reveal that:

-The polaronic effect causes a red-shift of the peak position of the optical response.

- The polaronic effect is enhanced with the decreasing quantum dot radius and that the increase in the radius leads also to a red-shift of the coefficients.

- The polaronic effect acts, as an enhancer of the electric dipole polarization and, in consequence, of the amplitude of the calculated optical properties.

\section{References}

1. T. H. Hood, J. Lightwave Technol. 6, 743 (1988).

2. I. Gerdova and A. Hache, Optics Communications 246, 205 (2005).

3. I. Moreels, P. Kockaert, R. Van Deun, K. Driesen, J. Loicq, D. Van Thourhout, and Z. Hens, J. Lumin. 121, 369 (2006).

4. H. Yildirim and M. Tomak, J. Appl. Phys. 99, 093103 (2006). 
5. L. Zhang, Superlattices Microstruct. 37, 261 (2005).

6. R. Turton, The Quantum Dot: A Journey into the Future of Microelectronics (Oxford University Press, New York, 1995).

7. G. Bastard, J. A. Brum, and R. Ferreira, Solid State Phys.44, 229 (1991).

8. N. P. Montenegro and S. T. PerezMerchancano, Phys. Rev. B 46, 9780 (1992).

9. I. Karabulut and S. Baskoutas, J. Appl. Phys. 103,1073512 (2008).

10. J. M. Ferreyra and C. R. Proetto, Phys. Rev. $B$ 52, R2309 (1995).

11. J. M. Ferreyra, P. Bosshard, and C. R. Proetto, Phys. Rev. B 55, 13682 (1997).

12. C. Bose and C. K. Sarkar, Solid-State Electron. 42, 1661 (1998).

13. S. Baskoutas and A. F. Terzis, Eur. Phys. J. B 69,237 (2009).

14. S. Baskoutasa, A. F. Terzis, Physica E 40, 1367(2008).

15. J. L. Zhu and X. Chen, Phys. Rev. B 50, 4497 (1994).

16. S. S. Li and J. B. Xia, Phys. Lett. A 366, 120 (2007).

17. A. Ibral, A. Zouitine, E. M. Assaid, E. Feddi and F. Dujardin, Physica B 449, 261 (2014).

18. D. B. Hayrapetyan, E. M. Kazaryan, L. S. Petrosyan, H. A. Sarkisyan, Phys. E 66, 7 (2015).

19. G. H. Wang, Optics Communications. 355, 1 (2015).

20. S. Mzerd, M. El Haouari, M. Aghoutane, M. El-Yadri, E. Feddi, F. Dujardin, I. Zorkani, A. Jorio, M. Sadoqi, and G. Long, Journal of Applied Physics 124, 164303 (2018).

21. E. M. Goldys and J. J. Shi, Phys. Status Solidi B 210, 237 (1998).

22. D. Ahn and S. L. Chuang, IEEE J. Quantum Electron. 23, 2196 (1987).

23. I. Karabulut, U. Atav, H. Safak, and M. Tomak, Eur. Phys. J. B 55, 283 (2007).

24. Y. Kan, H. Nagae, M. Yamanishi, and I, Suemune, IEEE J.Quantum Elect. 23, 2167 (1987)
25. K. J. Kuhn, G. U. Lyengar, and S. Yee, J. Appl. Phys. 70,5010 (1991).

26. R. F. Kazarinov and R. A. Suris, Sov. Phys. Semicond.5, 707 (1971).

27. D. A. B. Miller, Int. J. High Speed Electron. Syst. 1, 19 (1991).

28. J. J. Shi, S. H. Pan, Superlattice Microstruct. 17,91 (1995).

29. S. Unlu, I Karabulut, H. Safak, Physica E.33,319 (2006) .

30. C. H. Liu, B. R. Xu, Phys. Lett. A 33, 324 (2006)

31. A. L. Vartanian,L. A. Vardanyan, E. M. Kazaryan, Phys. Lett. A 360, 649 (2007).

32. A. L. Vartanian, L. A. Vardanyan, E. M. Kazaryan, Phys. Status Solidi B 245, 123 (2008).

33. A. L. Vartanian, L. A. Vardanyan, E. M. Kazaryan, Physica E 40, 1513 (2008).

34. Y. F. Huangfu, Z. W. Yan, Physica E 40, 2982 (2008)

35. K. M. Kumar, A. J. Peter, C. W. Lee, Superlattices and Microstruct.51, 184 (2012) .

36. L. Shi, Z. W. Yan, J. Appl. Phys. 110, 024306 (2011).

37. L. Shi, Z. W. Yan, Eur. Phys. J. B 86, 244 (2013).

38. $\quad$ L. Shi, Z. W. Yan, Solid State Commun. 209210, 27(2015).

39. S. M'zerd, M. El Haouari, A. Talbi, E. Feddi , M.E.Mora-Ramos, Journal of Alloys and Compounds 753 (2018) 68e78.

40. H. Frohlich, Proc. R. Soc. Edinburgh, Sect. A: Math. 215, 291 (1952).

41. T. D. Lee, F. Low and D. Pines, Phys. Rev. 90, 297(1953).

42. C. Aldrich and K. K. Bajaj, Solid State Commun.22, 157 (1977)

43. M. Kirak, S. Yilmaz, M. Sahin, M. Gencaslan, J. Appl. Phys. 109, 094309 (2011). 